\title{
LncRNA SNHG1 and RNA Binding Protein hnRNPL form a Complex and Coregulate CDH1 to Boost the Growth and Metastasis of Prostate Cancer
}

\section{Xiao Tan}

The Affiliated Hospital of Southwest Medical University

\section{Wen-Bin Chen}

Zhujiang Hospital

Dao-Jun Lv

Guangzhou Medical College First Affiliated Hospital

\section{Tao-Wei Yang}

Zhujiang Hospital

\section{Kai-Hui Wu}

Zhujiang Hospital

\section{Li-Bin Zou}

Zhujiang Hospital

Junqi Luo

Zhujiang Hospital

\section{Xu-Min Zhou}

Zhujiang Hospital

\section{Guo-Chang Liu}

Guangzhou Women and Children's Medical Center

\section{Fang-Peng Shu}

Guangzhou Women and Children's Medical Center

Xiangming Mao ( $\nabla$ mxm@smu.edu.cn )

Zhujiang Hospital

\section{Research}

Keywords: Heterogeneous nuclear ribonucleoprotein L (hnRNPL), Small Nucleolar RNA Host Gene 1 (SNHG1), E-cadherin (CDH1), Epithelial-mesenchymal transition (EMT), prostate cancer (PCa)

Posted Date: October 2nd, 2020

DOl: https://doi.org/10.21203/rs.3.rs-84130/v1 
License: (c) (i) This work is licensed under a Creative Commons Attribution 4.0 International License. Read Full License 


\section{Abstract}

Background: The interaction between LncRNA and RNA-binding protein (RBPs) plays an essential role in the regulation over the malignant progression of tumors. Previous studies on the mechanism of SNHG1, an emerging IncRNA, have primarily focused on the competing endogenous RNA (ceRNA) mechanism. Nevertheless, the underlying mechanism between SNHG1 and RBPs in tumors remains to be explored, especially in prostate cancer ( $\mathrm{PCa}$ ).

Methods $\llbracket S N H G 1$ expression profiles in PCa were determined through the analysis of TCGA data and tissue microarray at the mRNA level. Gain- and loss-of-function experiments were performed to investigate the biological role of SNHG1 in PCa initiation and progression. RNA-seq, immunoblotting, RNA pull-down and RNA immunoprecipitation analyses were utilized to clarify potential pathways with which SNHG1 might be involved. Finally, rescue experiments were carried out to further confirm this mechanism.

Results: We found that SNHG1 was dominantly expressed in the nuclei of PCa cells and significantly upregulated in PCa patients. The higher expression level of SNHG1 was dramatically correlated with tumor metastasis and patient survival. Functionally, overexpression of SNHG1 in PCa cells induced epithelial-mesenchymal transition (EMT), accompanied by down-regulation of the epithelial marker, $E$ cadherin, and up-regulation of the mesenchymal marker, vimentin. Increased proliferation and migration, as well as accelerated xenograft tumor growth, were observed in SNHG 1-overexpressing PCa cells, while opposite effects were achieved in SNHG 1-silenced cells. Mechanistically, SNHG1 competitively interacted with $h n R N P L$ to impair the translation of protein E-cadherin, thus activating the effect of SNHG1 on the EMT pathway, eventually promoting the metastasis of PCa.

Conclusion: Our findings demonstrate that $S N H G 1$ is a positive regulator of EMT activation through the SNHG1-hnRNPL-CDH1 axis. SNHG1 may serve as a novel potential therapeutic target for PCa.

\section{Background}

Prostate cancer (PCa)is the frequency life-threatening tumor in male genitourinary system[1]. The malignant transformation of the prostate follows a multi-step process, starting with prostatic intraepithelial neoplasia (PIN), Then there is localized prostate cancer, followed by locally invasive advanced prostatic adenocarcinoma, which eventually develops into metastatic $\mathrm{PCa}$ [2]. The patient eventually developed metastatic castration resistant prostate cancer (mCRPC) due to castration resistance and metastasis, resulting in death. There's growing evidence that epithelial-mesenchymal transition (EMTDemerges a great role in the metastasis of various cancers, including $\mathrm{PCa}[3-5]$. The progression of $\mathrm{PCa}$ is closely related to the proliferation and invasion phenotype of cancer cell [6]. However, the cellular and molecular mechanisms responsible for this metastasis PCa are incompletely understood.

Long non-coding RNAs (LncRNAs) are RNAs with a length of more than 200 nucleotides without proteincoding function. In recent years, the expansion of knowledge has revealed that IncRNAs play an 
important role in multiple biological processes, such as alternative splicing, nuclear import, imprinting, cell differentiation and RNA decay[7]. The abnormal expression of IncRNAs have also been reported to contribute to tumorigenic processes of many human malignancies, including $\mathrm{PCa}[8,9]$. In $\mathrm{PCa}$, recent studies have revealed that LncRNA DLX6-AS1 enhances PCa Malignant Phenotype and Lymph Node Metastasis[10], and LncRNA AC245100.4 promotes the proliferation of PCa through binding to HSP90[11]. Small nucleolar RNA host gene 1(SNHG1), a novel LncRNA, has shown to be aberrantly high expression and oncogenic characteristics in various cancers[12]. Previous studies have illustrated that SNHG1 was upregulated in PCa and was associated with PCa proliferation through the namely competing endogenous RNA (ceRNA) mechanism $[13,14]$. While, the specific function of SNHG1 have not been well investigated in the context of PCa metastasis and the underlying mechanism are also needed to be elucidated.

In this study we sought to determine the expression and the biological function of SNHG1 in PCa, especially its role in metastasis. Expression levels of $S N H G 1$ were determined in the PCa primary tumor tissues and its correlations with clinicopathological parameters were also analyzed. We further investigated the effects of SNHG1 on the aggressive phenotypes of PCa cell lines in vitro and in vivo. The regulatory role of $S N H G 1$ on $C D H 1$ were also explored to elucidate the potential mechanisms. Taken together, our results have demonstrated that $S N H G 1$ played a critical role in the progression of PCa.

\section{Methods}

\section{Patients and clinical samples}

Formalin fixed paraffin - embedded (FFPE) prostate cancer specimens were obtained from patients that undergo radical prostatectomy (RP) at zhujiang hospital from 2015 to 2018. The pathological types of the control group (total 14 specimens) were benign prostatic hyperplasia (BPH) or adjacent prostatic tissues. Patients' clinical information was obtained by reviewing the follow-up records of their electronic medical records. The median age of the enrolled patients was 67.5 years and average age was 63.3 (range: 22-82 years). Clinical TNM staging and Gleason scores of patient specimens were based on the American Joint Committee on Cancer (AJCC) Eighth Edition (2017) and the 2016 World Health Organization (WHO) classification of genitourinary tumors. The detailed clinicopathological information of all samples was presented in TableS1. All patients agreed to participate in this study by signing an informed consent in accordance with the ethical scheme formulated by the ethics committee of Zhujiang Hospital, Southern Medical University.

\section{Survival Analysis}

Association of LncRNA SNHG1 and $h n R N P L$ level on clinical events was evaluated using The Cancer Genome Atlas (TCGA) prostate adenocarcinoma cohort. Survival and expression data were acquired from TCGA data portal. Gene Expression Profiling Interactive Analysis (GEPIA) was utilized to analyze Overall survival and disease-free survival[15]. Statistical significance was evaluated by Kaplan-Meier analysis and log-rank test. 


\section{Fluorescence in situ hybridization (FISH)}

Fluorescence in situ hybridization was constructed to identify the intracellular expression and sublocalization of SNHG1 according to the protocol described before[16], with minor modifications. Prostate cancer cells were grown on $15 \mathrm{~mm}$ confocal dishes (JET BIOFIL, Guangzhou, China). Probe sequences targeting homo-SNHG1 were designed and generated by Ribo Bio(Guangzhou, China). Positive control probe sequences targeting homo-18S (cy3, Ribo Bio, Guangzhou, China), All operations were carried out in accordance with the Ribo ${ }^{\mathrm{TM}}$ Fluorescent In Situ Hybridization kit instructions, and minor modifications were made during the prostate tissue FISH assay. The samples were captured in Olympus laser scanning microscope FV3000 (Olympus Corporation, Japan). Images were processed in ImageJ through 3 major steps: background subtraction, set a threshold to label all the cells and label the SNHG1 positive cells.

\section{Cell Culture And Cell Lines}

We used five human prostate cancer cell lines and one immortalized prostatic epithelial cell line, all the cells including RWPE-1, LNCaP, 22Rv1, PC-3, DU145 were obtained from Stem Cell Bank, Chinese Academy of Sciences. RPMI-1640 medium containing 10\% fetal bovine serum (FBS, Hyclone) was used to culture all prostate cancer cells, and the Keratinocyte Serum Free Medium (KSFM) (Gibco, No. 10744019) added in $5 \mathrm{ng} / \mathrm{mL}$ epidermal growth factor (EGF) (Gibco, No. 10450-013) was suitable for culturing RWPE- 1 cells. A Wild environment $\left(37^{\circ} \mathrm{C}\right.$ in $\left.5 \% \mathrm{CO}_{2}\right)$ was set for maintaining all cells.

\section{Construction of cells with stable knockdown of SNHG1}

We designed and synthesized three small - interfering RNAs targeting SNHG1 (NR_003098.2) and negative control (NC) siRNA negative control sequence without a specific target, all the siRNAs were synthesized by GeneChem (Shanghai, China). The sequence of siRNAs targeting SNHG1 is as follows, sih-SNHG1_001: CAGCA GTTGA GGGTT TGCTG TGTAT; si-h-SNHG1_002: TTCAA CAGCT AGGTT GTCCTT; si-h-SNHG1_003: GACCU AGCUU GUUGC CAAUTT. SNHG1 overexpressed cells used pcDNA3.1 system, which was synthesized by VigeneBio co. (Shandong, China).In this study, we used lentiviral vectors (GeneChem, Shanghai, People's Republic of China) to construct stable silencing SNHG1 PCa cell lines following to the authoritative manufacturer's instruction. Target sequence of shRNA (short hairpin RNA) againsting SNHG1 was 5'-GACCU AGCUU GUUGC CAAU-3'. Stable SNHG1 silenced cell lines sh-SNHG1 were kept in $5 \mu \mathrm{g} / \mathrm{mL}$ puromycin for 10 days and then confirmed that it's Validity and specificity.

\section{Quantitative RT-PCR assay and RNA extraction}

The RNAiso Plus reagent (TaKaRa) and PrimeScript RT reagent Kit (TaKaRa) were utilized to extract total RNA and generate cDNA according to the official protocols. Extraction of nuclear and cytoplasmic RNA utilized the Ambion ${ }^{\circledR}$ PARIS ${ }^{\mathrm{TM}}$ system according to the kit procedures. The quantitiative reverse transcriptase-PCR (qRT-PCR) reactions was performed with the SYBR Green PCR Master Mix (TaKaRa) with ABI7500 Fast Real-Time RCR System (Applied Biosystems, USA). The Primer3 system was utilized to 
design the gene unique primers which were subsequently synthesized through TsingKe Biological Technology (Guangzhou, China). Every survey was conducted in three times, and GAPDH was utilized to normalize the results as internal reference. The relative quantification method which calculated by $2^{-\Delta \Delta C t}$ was used in analyzing the expression data.

\section{CCK-8 assay}

2,000 PCa cells were seeded per well in 96-well plates, and Each well contained medium containing $10 \%$ FBS with a total volume of $100 \mathrm{ml}$. According to the counting kit-8 (CK-04, Dojindo) manufacturer's operating instructions, after the cells were adherent to the plates, the original medium was removed, RPMI1640 diluted CCK-8 reagent (10\% v/v) in $100 \mu \mathrm{l}$ was added to the cells, following 2 hours of incubated. Then, the optical density (OD) at $450 \mathrm{~nm}$ was measured by a microplate reader (EXL800, BioTek Instruments).

\section{Colony Formation}

PCa cells were inoculated into 6 - pore plates at a rate of 500 cells per well in a $2 \mathrm{~mL}$ medium containing $10 \%$ fetal bovine serum, and incubated for two weeks for colony formation analysis. Then, $4 \%$ paraformaldehyde were used to fix the colonies and Giemsa was applied to stain colonies. The experiment of colony formation was repeated three times with three Wells in each group.

\section{EdU incorporation Assay}

Cell-Light EdU staining kit (RiboBio, Guangzhou, China) was utilized to detect cell proliferation activity accroding to the manufacturer's instructions. Images were obtained by a fluorescence microscope (Olympus, Tokyo, Japan) at 200x. The proportion of EdU positively stained cells to Hoechst-stained cells (with blue fluorescence) in per well was equal to the cell proliferation rate.

\section{Transwell Migration Assay}

Transwell (Costar, Corning, USA) with a membrane of numerous pole $(8.0 \mu \mathrm{m})$ was used to perform Cell migration assay. 35000 cells were hybridized in FBS-free RPMI-1640 and sowed into the upper chamber of the well. Then, $500 \mu \mathrm{L}$ complete medium with FBS was put into the sublayer. After 24 hours, $4 \%$ paraformaldehyde and Giemsa (Boster Ltd., Wuhan, China) were used to fix and stain the cells. After that, wiping out the cells on the top surface of the membrane, and the cells on the bottom surface were photographed through an inverted microscope. (Olympus DP72). Five randomly visual fields were chosed to count the cell number by using Image $\mathrm{J}$ software.

\section{Mice xenograft analysis}

Xenograft models were created through persistent injection of $5 \times 10^{6}$ DU145/sh-NC, DU145/sh-SNHG1 cells ( $n=7$ per group), on the axillae of nude male mice (4-5 weeks). Tumor sizes were measured twice a week and formula 
was used to calculate tumor volume. Mice were raised in Specific Pathogen Free (SPF) environments for 4 weeks. Subsquently, tumor samples were carefully resected, photographed and specimens were further investigated by hematoxylin and eosin(H\&E) and IHC. The Animal Care and Use Committee of Southern Medical University approved all our experimental animal programs.

\section{Antibodies and Western blot analyses}

Related cell lysates were run on 4-12\% SDS PAGEs to conduct Western blot analyses. RIPA lysis buffer containing protease inhibitors (\#KGP250, KeyGEN BioTECH, Nanjing, China) was used to extracted PCa cells protein following to the operation protocol. Then, equal amounts of 30ug proteins were separated by SDS/PAGEs and transferred onto PVDF membranes (Millipore, Billerica, MA, USA) electrically. Then, membranes were blocked with TBS(Tris/saline solution with $0.1 \%$ Tween-20) including $5 \%$ milk without fat for $1 \mathrm{~h}$ and incubated overnight at $4{ }^{\circ} \mathrm{C}$ covering by unique antibodies: rabbit anti- $\beta$-actin (\#4970, CST), rabbit anti-Slug (\#9585), rabbit anti-ZEB1(\#3396, CST), rabbit anti-E-Cadherin (\#3195, CST) and rabbit anti-hnRNPL (4D11, \#ab6106, Abcam). All membranes were subsequently incubated at room temperature for 1 hour with horseradish peroxidase-linked secondary anti-rabbit or anti-mouse IgG antibodies (Cell Signaling Technology). Final bands were visualized by ECL kit ((Pierce Biotechnology, Rockford, IL, USA). Image $J$ was applied to quantify the intensity of the band

\section{Plasmid transfection}

The full-length wild-type human SNHG1 and 401-607bp truncated mutant SNHG1-mut were inserted into pcDNA3.1 expression vector acquired from Shangdong Vigene Biosciences Co. All constructs were confirmed by sequencing. Referring to the Lipofectamine ${ }^{\mathrm{TM}} 3000$ Transfection Reagent instructions (L3000015, Thermo Fisher Scientific, USA), DU145/sh-SNHG1 cell line was transiently transfected with SNHG1-mut or SNHG1, DU145 and C4-2 cells were transfected with SNHG1 or meaningless vector. Expression of Plasmid was confirmed by qRT-PCR.

\section{Immunohistochemical analysis and assessment}

Prostate tissue $\mathrm{IHC}$ were employed to investigate protein expression in tissues as described previously. Relative proteins expression was investigated in Prostate tissue IHC as previously referred to[17]. In IHC,

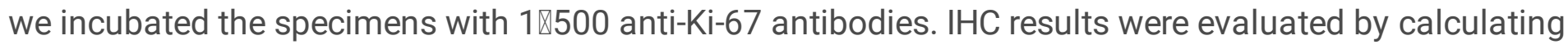
the percentage of positive nuclei, the percentage of positive cells and the staining concentration. Every assessment was carried out by three separated advanced pathologists utilizing the parallel microscope.

\section{RNA-seq processing}


RNA sequencing and sequence quality control was performed by BGISEQ platform. Human genome reference was established from UCSC version GRCh38/hg38 chromosomes 1-22, X, Y and mitochondrial DNA. The further analysis including heatmap, gene set enrichment analysis, and gene annotation of sequencing data was completed by BGI Dr. Tom system[18].

\section{RNA-protein pulldown and RNA immunoprecipitation (RIP)}

In vitro biotin-labeled RNAs (SNHG 1, AR-3'UTR and Ploy $(\mathrm{A})_{25}$ ) and RNA-protein pull-down assay were done with Pierce ${ }^{\mathrm{TM}}$ Magnetic RNA-Protein Pull-Down Kit (\#20164, Thermo Scientific $\left.{ }^{\mathrm{TM}}\right)$. Pull-down protein samples were ran on SDS-PAGE gels, following silver staining to Identify the difference bands, then the difference bands were analyzed by mass spectrometry. RIP assays were performed by utilizing EZ-Magna RIP $^{\text {TM }}$ Kit (\#17-701, Merck Millipore) following the protocol.

\section{In silico binding prediction}

To obtain potential SNHG1 and $h n R N P L$ binding sites, we first predicted the interaction probability between SNHG1 and hnRNPL sequences in RNA-Protein Interaction Prediction(RPISeq)[19]. Furthermore, catRAPID was used to predict the binding site of hnRNPL on SNHG1[20]. The highest-raking site at RNA position 401-677bp includes CA-rich motifs and thus used for subsequent analysis.

\section{Statistical analysis}

Statistical analyses were carried out with GraphPad Prism8 (GraphPad Software, La Jolla, USA). Student's $t$ test or chi-square test was applied to determine the statistical significance of differences between distinct groups, if proper. All results were expressed as mean \pm standard deviation (SD), and the two-tailed $p$ value of 0.05 was considered statistically significant.

\section{Results}

\section{LncRNA SNHG1 is upregulated in prostate cancer tissues and sub-located in the nucleus}

To identify the expression and localization of SNHG1 in PCa, we utilized bioinformatics to analyze the expression and prognosis of SNHG1 in PRAD in TCGA database. As shown in (Fig. 1A-C), we identified $S N H G 1$ transcript level is overexpression in prostate cancer. Meanwhile, we evaluated the correlation between SNHG1 level and clinical outcomes from TCGA database using Kaplan-Meier analysis and logrank tests. In the PRAD group, the prognosis of 247 PCa patients with high transcriptional level of SNHG1 was significantly worse than that of 248 patients with low expression of SNHG1.

Next,the expression and localization of $S N H G 1$ was validated in 67 prostate cancer tissues and 14 adjacent normal tissues via RNA FISH. we divided the sample into high $(++,+++)$ and low $(-,+)$ SNHG1 expression group according to the positive cell nuclear stain percentage of $S N H G 1$ and then explored the correlation between the clinicopathological characters of prostate cancer patients and SNHG1 expressions. SNHG1 was significantly overexpressed in $67.16 \%$ (45 of 67 PCa tissues) and low 
expression in all(14 of 14 normal adjacent tissues) normal samples and $32.84 \% \varangle 22$ of 67 PCa tissues (Table 1 and Fig. 1D). Besides $\square$ as shown in Table 1, SNHG1 transcript level was positively associated with Gleason Score but It may not be related to the patient's age and clinical TNM stage in our study by Chisquare tests.

Table 1

Expression of SNHG1 in normal prostate tissues and prostate cancer tissues.

\begin{tabular}{|c|c|c|c|c|c|c|}
\hline & \multirow[t]{2}{*}{ Group } & \multirow[t]{2}{*}{$\mathrm{N}$} & \multicolumn{2}{|c|}{ SNHG1 expression } & \multirow{2}{*}{$x^{2}$} & \multirow[t]{2}{*}{$\mathrm{p}$-Value } \\
\hline & & & low & high & & \\
\hline \multirow[t]{2}{*}{ Type } & Normal & 14 & 14 & 0 & \multirow[t]{2}{*}{21.157} & \multirow[t]{2}{*}{$<0.001$} \\
\hline & Adenocarcinoma & 67 & 22 & 45 & & \\
\hline \multirow[t]{2}{*}{ Age } & $\leq 65$ & 26 & 8 & 18 & \multirow[t]{2}{*}{0.082} & \multirow[t]{2}{*}{0.774} \\
\hline & $>65$ & 41 & 14 & 27 & & \\
\hline \multirow[t]{2}{*}{ Clinical stage } & $\mathrm{I}-\mathrm{II}$ & 42 & 15 & 27 & \multirow[t]{2}{*}{0.423} & \multirow[t]{2}{*}{0.516} \\
\hline & III-IV & 25 & 7 & 18 & & \\
\hline \multirow[t]{2}{*}{ Primary tumor } & $\mathrm{T} 1-\mathrm{T} 2$ & 44 & 16 & 28 & \multirow[t]{2}{*}{0.723} & \multirow[t]{2}{*}{0.395} \\
\hline & T3-T4 & 23 & 6 & 17 & & \\
\hline \multirow[t]{2}{*}{ Gleason score } & $\leq 6$ & 17 & 9 & 8 & \multirow[t]{2}{*}{4.175} & \multirow[t]{2}{*}{0.041} \\
\hline & $\geq 7$ & 50 & 13 & 37 & & \\
\hline
\end{tabular}

SNHG1 expression was determined by FISH; $p$-value is from $\chi^{2}$-test -test.

A remarkably increasing frequency of positive expression of SNHG1 was detected in prostate cancer specimens compared to normal prostate tissues $(P<0.001, \chi 2$-test).

Furthermore, we validated the higher expression level of SNHG1 in DU145, PC3, LNCaP, c4-2 and other prostate cancer cell lines compared to that in RWPE-1 cell line (Fig. 1E). Then, we cultured prostate cancer cell DU145 and C4-2 as cell models of high and low SNHG1 expression in prostate cancer. Through cellular fractionation assays (Fig. 1F) and RNA fluorescence in situ hybridization(FISH)(Fig. 1G),we demonstrated that SNHG1 was mainly distributed in the nucleus of DU145 cells and cytoplasm of C4-2 cells. Taken together, our data confirmed that $S N H G 1$ is highly expressed in prostate cancer tissues and cell lines,which might potentially serve as a novel independent predictor of overall survival in prostate cancer.

LncRNA SNHG1 is essential for promoting PCa cells proliferation, migration in Vitro 
To figure out the potential role of $S N H G 1$ in promoting prostate cancer progression,we took advantage of siRNA or lentivirus shRNA to knock down the endogenous expression of SNHG1 in DU145 and C4-2 cells and used an overexpression plasmid system to upregulate SNHG1 in DU145 and C4-2 cells. CCK-8 assays, colonies formation and EDU assays demonstrated that upregulated SNHG1 remarkably enhanced PCa cells proliferation, while SNHG1 knockdown significantly blocked the proliferative abilities of Prostate cancer cells (Fig. 2A-C). Notably, SNHG1 knockdown impaired the migration ability of PCa cells, while upregulated SNHG1 transcription level promoted cell migration as the results revealed (Fig. 2D-E). These results suggested that $S N H G 1$ promotes cell proliferation, migration in PCa cells.

\section{Knockdown LncRNA SNHG1 inhibited PCa cells tumorigenesis in vivo}

For the purpose of validating the potential impact of SNHG1 depletion on the tumorigenesis of PCa in vivo, DU145 cells transfected with sh-SNHG1 or Negative Control lentivirus vector were injected subcutaneously in male nude mice. Tumors in mice implanted in DU145/sh-SNHG1 cells were remarkably smaller than those in the control cells (Fig. 3A-B). Likewise, SNHG1 knockdown cells grew slower in compared with the control cohort, the average tumor volumes and weight in the ultimate experiment were significantly lower in the DU145/sh-SNHG1 cohort versus the negative control lentivirus vector cohort (Fig. 3C-D). IHC staining of ki-67 antigen confirmed that subcutaneous tumor proliferation was significantly reduced in the sh-SNHG1 group (Fig. 3E).

\section{Related genes of IncRNA SNHG1 in PCa cells.}

To validate the potential related genes that may be regulated by SNHG1 in PCa cells, RNA transcriptome sequencing was carried out in DU145/NC or DU145/sh-SNHG1 cells. As a result of silencing SNHG1, a total of 837 genes greater than 1.5-fold change increased, whereas 208 genes exhibited a decrease in abundance (<-1.5 fold change) (Fig. 4A). Meanwhile, A thorough study of the oncology analysis highlighted the most obvious biological phenomena of overexpression associated with the pathways in cell adhesion molecules (CAMs) (Fig. 4B-D), and the RNA sequencing data were verified by qRT-PCR (Fig. 4E). However, the mechanism by which SNHG1 regulates CAMs is still unclear.

\section{SNHG1 activates the EMT pathway in PCa cells through interaction with hnRNPL}

In order to further clarify the pathway of SNHG1 regulating network, we used RNA-protein pull-down to analyze the protein molecules interacting with SNHG1. By silver staining of pull-down protein SDS-PAGE gel, we found that $S N H G 1$ was specifically bound to $60-70$ kda protein bands (Fig. 4F), the main protein of SNHG1 interaction was identified as hnRNPL by mass spectrometry and WB (Fig. 4G-I). Besides, the interaction between $h n R N P L$ and SNHG 1 was further predicted by RPISeq (Fig. 4H), and the binding relationship was double checked by $h n R N P L$ RIP-PCR (Fig. 4J). Furthermore, western blot showed that SNHG1 knockdown and overexpression did not affect the hnRNPL protein level, and it can regulate EMT pathway (Fig. 4K-L). Finally, our previous work confirmed that LncRNA SNHG1 triggers EMT pathway through directly binding to $h n R N P L$ in the development of prostate cancer. 
Next, to investigate the exact binding site between $S N H G 1$ and $h n R N P L$, CatRAPID fragment prediction was used to reveal that the sequence of $S N H G 1$ at 400-600 bp have a significant binding possibility with hnRNPL (Fig. 5A). Then, we constructed SNHG1-mut truncated mutant overexpression vector with 400607 bp deletion of SNHG1 sequence compared with the SNHG1-wt (Fig. 5B). In DU145/sh-SNHG1 cells, Vector, SNHG1-mut and SNHG1-wt plasmids were transfected, and the over-expression efficiency of plasmids was verified by qRT-PCR (Fig. $5 \mathrm{C}$ ). Meanwhile, the results of $h n R N P L$ RIP-PCR in aforesaid cells confirmed that 400-607 bp truncated SNHG1-mut could not bind to hnNRPL protein, In addition, hnRNPL also lost the ability to capture $\mathrm{CDH} 1$ to a great extent when it could not specifically bind to SNHG1 compared with SNHG1-wt overexpressed cell lines (Fig. 5D). Meanwhile, Transwell and wound healing assays demonstrated that overexpressing mutant SNGH1 could not promote cell migration ability in PCa cells, when compared with the wild type SNHG1 (Fig. 5E-F). As hnRNPL is essential for mRNA stability, we wondered if the SNHG1/hnRNPL stabilizes certain unknown downstream effector proteins, such as Ecadherin. Therefore, using siRNA targeting on $h n R N P L$, we effectively inhibited its expression in DU145 cells. Surprisingly, we found that E-cadherin was remarkbly downregulated both in mRNA and protein levels, while SNHG1 did not show obvious difference (Fig. 5G-H). We further found that knockdown of hnRNPL significantly reduced the mRNA stability of $C D H 1$ (E-cadherin) (Fig. 5I). Therefore, we found that SNHG1/hnRNPL complex could break the balance between hnRNPL and E-cadherin mRNA, causing to Ecadherin protein downregulation.

\section{HnRNPL knockdown reverses the carcinogenic effects of SNHG1 promotion on cell metastasis}

To explore whether SNHG1 exerts its biological function by binding to $h n R N P L$, a rescue assay between $S N H G 1$ and $h n R N P L$ was performed. Transwell and wound healing assays revealed that $h n R N P L$ knockdown significantly reversed the SNHG1 overexpression-induced cell migration ability in PCa cells (Fig. 6A-B). Meanwhile, western blot showed that forced expression of hnRNPL reversed the SNHG1 upregulating-induced inhibition of E-cadherin protein (Fig. 6C). Moreover, Survival analysis revealed that PCa patients with both low SNHG1 and HnRNPL expression levels had better overall survival and diseasefree survival with GEPIA (Fig. 6D). In general, we illustrated that LncRNA SNHG1 contributed to PCa growth and metastasis by directly binding to hnRNPL to coregulate E-cadherin, which activates PCa EMT (Fig. 6E).

\section{Discussion}

In recent years, the incidence of prostate cancer has been on the rise in China. Despite that the diagnosis rate of prostate cancer has been improved by diagnostic methods such as screening PSA and imaging, the mortality caused by prostate cancer remains unchanged, and metastasis is a critical factor leading to the death of prostate cancer[21,22]. Therefore, to reveal the pathogenesis of prostate cancer may help us find specific biomarkers, which bears great significance for the diagnosis, treatment and prognosis of patients with prostate cancer. 
LncRNAs participates in the development of cancer through a variety of mechanisms that regulate transcription, translation and protein function[23]. LncRNA prostate cancer antigen 3 (PCA3) has been approved by the U.S. FDA as a marker for the diagnosis of prostate cancer, and has also been included in "Guidelines for Diagnosis and Treatment of Urological Diseases in China"[24], announcing tremendous potential of IncRNA as a biomarker for the diagnosis and treatment of prostate cancer, which is worthy of in-depth study. Although Several studies proved that SNHG1 promoted PCa proliferation via serving as "miRNA sponeges" [11, 12], the functions and potential mechanisms in PCa remains unclear. We identified IncRNA SNHG1 was overexpressed in prostate cancer tissues and cells, and associated with patient's clinical characteristics. Results of gain and loss of function shown that IncRNA SNHG1 promoted PCa cell proliferation and migration in vitro, and loss-of SNHG1 inhibited vivo tumor growth, suggesting that $S N H G 1$ is essential in PCa progression.

It is well known that LncRNAs mainly act as ceRNA to backout the inhibiting effects of miRNAs on their downstream targets[25, 26]. SNHG1 was also reported to regulate miR-199a-3p and miR-337-3p/AKT2 axis, leading to promotion in PCa cells $[11,12]$. However, recently studies revealed that RNA binding protein plays a crucial role in LncRNA regulating network. Peng et al. revealed that Lnc-FAM84B-4 interacts with protein hnRNPK to restrain MAPK pathway, leading to CRC progression[27]. Klingenberg et al. indicated that CASC9/ $h n R N P L$ serves as a IncRNA/protein complex associated with clinically relevant viability and affects AKT signaling in HCC[28]. Therefore, RNA-Pulldown and RIP was constructed to establish the SNHG1 and hnRNPL interaction in PCa cells. hnRNPL belongs to the hnRNP protein family, which participates in the malignant progression of tumors though mediating alternative splicing of precursor mRNA, strengthening mRNA stability, regulating mRNA translation etc [29-32]. Linked to our results, we demonstrated that the binding of $S N H G 1$ to $h n R N P L$ is responsible for a part of its cancerpromoting features in $\mathrm{PCa}$.

The EMT is a dynamic and critical process that enable the transition from early cancer to aggressive cancer, characterized via a decrease of epithelial markers and a upregulation in mesenchymal markers and EMT-related TFs[33], but the upper mechanisms regulating EMT require fully understanding. The major epigenetic events, such as DNA methylation, histone modification, nucleosome-reshaping, and noncoding RNAs, play vital roles in activaion or suppression of the initiation and sustainability of EMT[34-36]. CDH1, also known as E-cadherin, Notably, loss of E-cadherin was reckoned as the crucial step to initiate EMT that sustained PCa metastasis. Our works exhibited that the interaction of SNHG1 with $h n R N P L$ is responsible for a part of EMT through next generation sequencing and RIP assays in PCa cells. Furthermore, the $S N H G 1 / h n R N P L$ complex lead to a downstream of E-cadherin via binding to its mRNA, which promotes EMT in prostate cancer. These resluts also provide a new sight in SNHG1 regulating network.

Based on previous observations, we provide a strong evidence that $S N H G 1$ interacts with RNA-binding protein $h n R N P L$ to activate EMT pathway, promoting the malignant progression of $\mathrm{PCa}$, which is expected to provide a comprehensive understanding of the way SNHG1 acts on cancers. This thorough 
comprehension of the interaction relationship of $S N H G 1$ and $h n R N P L$ may lead to more valid strategies to diagnose and treat PCa.

\section{Conclusion}

In conclusion, we found that $S N H G 1$ promotes the proliferation and progression of $\mathrm{PCa}$, and high expression of both SNHG1 and hnRNPL can shorten the overall and disease-free survival of PCa patients. Further experiments demonstrated that SNHG1 can interact with $h n R N P L$ and ultimately leads to downregulation of E-cadherin expression and promote EMT progression, leading to tumor metastasis in PCa.

\section{Abbreviations}

PCa: prostate cancer; LncRNA: Long non-coding RNA; EMT: epithelial-mesenchymal transition; FBS: fetal bovine serum; PCR: polymerase chain reaction; qRT-PCR: Quantitative reverse transcription polymerase chain reaction; DAPI: 4', 6-Diamidino-2-phenylindole; TFs: transcription factors.

\section{Declarations}

\section{Acknowledgements}

Not applicable.

\section{Authors' contributions}

M.X.M, S. F. P and L.C.H conceived and designed the study. T.X, C.W.B, L.D.J carried out the experiments. Y. T. W, W. K.H and Z.L.B, collected and analyzed the clinical data. L.J.Q and Z.X.M analyzed and interpreted the data. C.W.B and L.D.J wrote the manuscript. M.X.M, S. F. P and L.D.J executed the funding acquisition. All authors have reviewed and approved the final version of the manuscript.

\section{Funding}

This work was financed by grants from the National Natural Science Foundation of China (No.81773277), Science and Technology Program of Guangzhou (No.201803010014), China Postdoctoral Science Foundation funded project (No.2018M643126 and 2019M662865), the Guangdong Basic and Applied Basic Research Foundation (No.2019A1515110033); Distinguished Young Talents in Higher Education Foundation of Guangdong Province (NO.2019KQNCX115); Achievement cultivation and clinical transformation application cultivation projects of the First Affiliated Hospital of Guangzhou Medical University (NO.ZH201908).

\section{Availability of data and materials}

All data generated or analyzed during this study are included in this published article. 


\section{Ethics approval and consent to participate}

All experiment protocols were approved by the Medical Ethics Committee of the Zhujiang Hospital Affiliated to Southern Medical University and performed following the World Medical Association Declaration of Helsinki. All procedures were approved by the Medical Ethics Committee of the Zhujiang Hospital Affiliated to Southern Medical University, and performed according to the Guide for the Care and Use of Laboratory Animals.

\section{Competing Interests}

The authors have claimed that no competing interest exists.

\section{Consent for publication}

All the patients signed written informed consent.

\section{References}

1. Siegel RL, Miller KD, Jemal A. Cancer statistics, 2020. CA: a cancer journal for clinicians. 2020.

2. Shen MM, Abate-Shen $\mathrm{C}$. Molecular genetics of prostate cancer: new prospects for old challenges. Genes \& development. 2010; 24: 1967-2000.

3. Kalluri R, Weinberg RA. The basics of epithelial-mesenchymal transition. The Journal of clinical investigation. 2009; 119: 1420-8.

4. Mittal V. Epithelial Mesenchymal Transition in Tumor Metastasis. Annual review of pathology. 2018; 13: 395-412.

5. Brabletz T, Kalluri R, Nieto MA, Weinberg RA. EMT in cancer. Nature reviews Cancer. 2018; 18: 128-34.

6. Body JJ, Casimiro S, Costa L. Targeting bone metastases in prostate cancer: improving clinical outcome. Nature reviews Urology. 2015; 12: 340-56.

7. Osielska MA, Jagodziński PP. Long non-coding RNA as potential biomarkers in non-small-cell lung cancer: What do we know so far? Biomedicine \& Pharmacotherapy. 2018; 101: 322-33.

8. Alahari SV, Eastlack SC, Alahari SK. Role of Long Noncoding RNAs in Neoplasia: Special Emphasis on Prostate Cancer. Int Rev Cell Mol Biol. 2016; 324: 229-54.

9. Prensner JR, Chinnaiyan AM. The emergence of IncRNAs in cancer biology. Cancer Discov. 2011; 1: 391-407.

10. Zhao Z, Liang S, Sun F. LncRNA DLX6-AS1 Promotes Malignant Phenotype and Lymph Node Metastasis in Prostate Cancer by Inducing LARGE Methylation. Front Oncol. 2020; 10: 1172.

11. Cui R, Liu C, Lin P, Xie H, Wang W, Zhao J, et al. LncRNA AC245100.4 binds HSP90 to promote the proliferation of prostate cancer. Epigenomics. 2020; 12: 1257-71.

12. Thin KZ, Tu JC, Raveendran S. Long non-coding SNHG1 in cancer. Clin Chim Acta. 2019; 494: 38-47. 
13. Li J, Zhang Z, Xiong L, Guo C, Jiang T, Zeng L, et al. SNHG1 IncRNA negatively regulates miR-199a$3 p$ to enhance CDK7 expression and promote cell proliferation in prostate cancer. Biochem Biophys Res Commun. 2017; 487: 146-52.

14. Xie M, Zhang ZY, Cui YP. Long Noncoding RNA SNHG1 Contributes to the Promotion of Prostate Cancer Cells Through Regulating miR-377-3p/AKT2 Axis. Cancer Biother Radio. 2020; 35: 109-19.

15. GEPIA . http://gepia.cancer-pku.cn/. Accessed 20 september 2020.

16. Raj A, van den Bogaard P, Rifkin SA, van Oudenaarden A, Tyagi S. Imaging individual mRNA molecules using multiple singly labeled probes. Nature methods. 2008; 5: 877-9.

17. Qiao H, Tan X, Lv DJ, Xing RW, Shu FP, Zhong CF, et al. Phosphoribosyl pyrophosphate synthetases 2 knockdown inhibits prostate cancer progression by suppressing cell cycle and inducing cell apoptosis. J Cancer. 2020; 11: 1027-37.

18. Multi-omics Interactivity system. http://biosys.bgi.com. Accessed 20 september 2020.

19. RNA-Protein Interaction Prediction (RPISeq). pridb.gdcb.iastate.edu/RPISeq/index.html. Accessed 20 september 2020.

20. catRAPID. http://service.tartaglialab.com/page/catrapid_group. Accessed 20 september 2020.

21. Siegel RL, Miller KD, Jemal A. Cancer statistics, 2018. CA Cancer J Clin. 2018; 68: 7-30.

22. Lughezzani G, Lazzeri M, Haese A, McNicholas T, de la Taille A, Buffi NM, et al. Multicenter European external validation of a prostate health index-based nomogram for predicting prostate cancer at extended biopsy. Eur Urol. 2014; 66: 906-12.

23. Yang GD, Lu XZ, Yuan LJ. LncRNA: A link between RNA and cancer. Bba-Gene Regul Mech. 2014; 1839: 1097-109.

24. Soares JC, Soares AC, Rodrigues VC, Melendez ME, Santos AC, Faria EF, et al. Detection of the Prostate Cancer Biomarker PCA3 with Electrochemical and Impedance-Based Biosensors. ACS Appl Mater Interfaces. 2019; 11: 46645-50.

25. Han D, Wang M, Ma N, Xu Y, Jiang Y, Gao X. Long noncoding RNAs: novel players in colorectal cancer. Cancer Lett. 2015; 361: 13-21.

26. Tay Y, Rinn J, Pandolfi PP. The multilayered complexity of ceRNA crosstalk and competition. Nature. 2014; 505: 344-52.

27. Peng W, Zhang C, Peng J, Huang Y, Peng C, Tan Y, et al. Lnc-FAM84B-4 acts as an oncogenic IncRNA by interacting with protein hnRNPK to restrain MAPK phosphatases-DUSP1 expression. Cancer Lett. 2020.

28. Klingenberg M, Gross M, Goyal A, Polycarpou-Schwarz M, Miersch T, Ernst AS, et al. The Long Noncoding RNA Cancer Susceptibility 9 and RNA Binding Protein Heterogeneous Nuclear Ribonucleoprotein L Form a Complex and Coregulate Genes Linked to AKT Signaling. Hepatology. 2018; 68: 1817-32.

29. Zhou X, Li Q, He J, Zhong L, Shu F, Xing R, et al. HnRNP-L promotes prostate cancer progression by enhancing cell cycling and inhibiting apoptosis. Oncotarget. 2017; 8: 19342-53. 
30. Lv D, Wu H, Xing R, Shu F, Lei B, Lei C, et al. HnRNP-L mediates bladder cancer progression by inhibiting apoptotic signaling and enhancing MAPK signaling pathways. Oncotarget. 2017; 8: 1358699.

31. Fei T, Chen Y, Xiao T, Li W, Cato L, Zhang P, et al. Genome-wide CRISPR screen identifies HNRNPL as a prostate cancer dependency regulating RNA splicing. Proc Natl Acad Sci USA. 2017; 114: E5207-E15.

32. Li Z, Chao T-C, Chang K-Y, Lin N, Patil VS, Shimizu C, et al. The long noncoding RNA THRIL regulates TNFa expression through its interaction with hnRNPL. Proc Natl Acad Sci USA. 2014; 111: 1002-7.

33. Nieto MA, Huang RY-J, Jackson RA, Thiery JP. EMT: 2016. Cell. 2016; 166: 21-45.

34. Skrypek N, Goossens S, De Smedt E, Vandamme N, Berx G. Epithelial-to-Mesenchymal Transition: Epigenetic Reprogramming Driving Cellular Plasticity. Trends Genet. 2017; 33: 943-59.

35. Sun L, Fang J. Epigenetic regulation of epithelial-mesenchymal transition. Cell Mol Life Sci. 2016; 73: 4493-515.

36. Serrano-Gomez SJ, Maziveyi M, Alahari SK. Regulation of epithelial-mesenchymal transition through epigenetic and post-translational modifications. Mol Cancer. 2016; 15: 18.

\section{Figures}


A

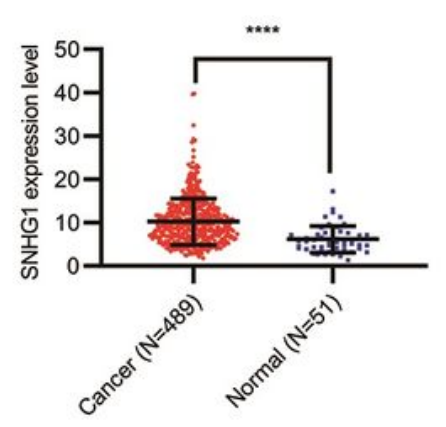

B

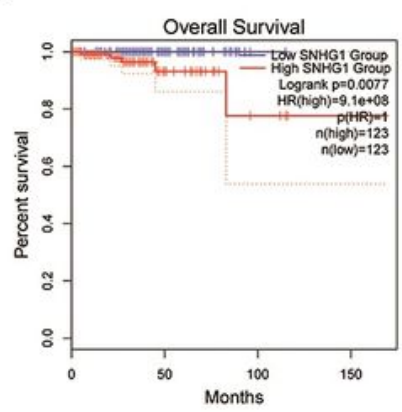

C

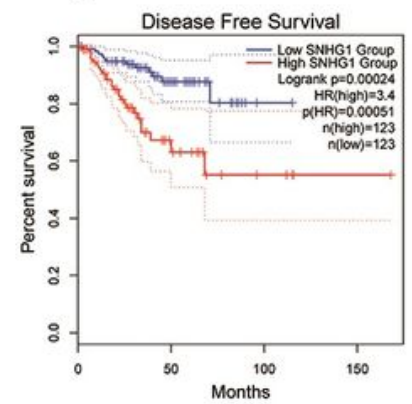

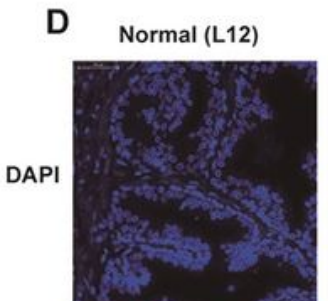

GS: $3+3$ (B8)

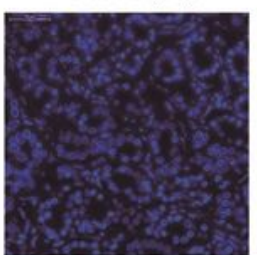

GS: $3+4(C 7)$

GS:4+4 (E4)
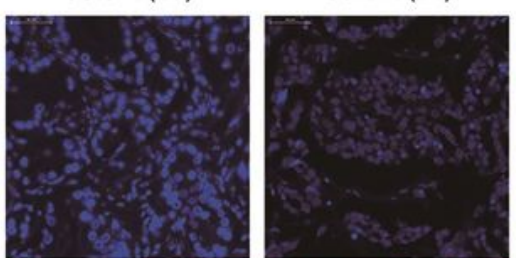

GS:5+5 (J16)
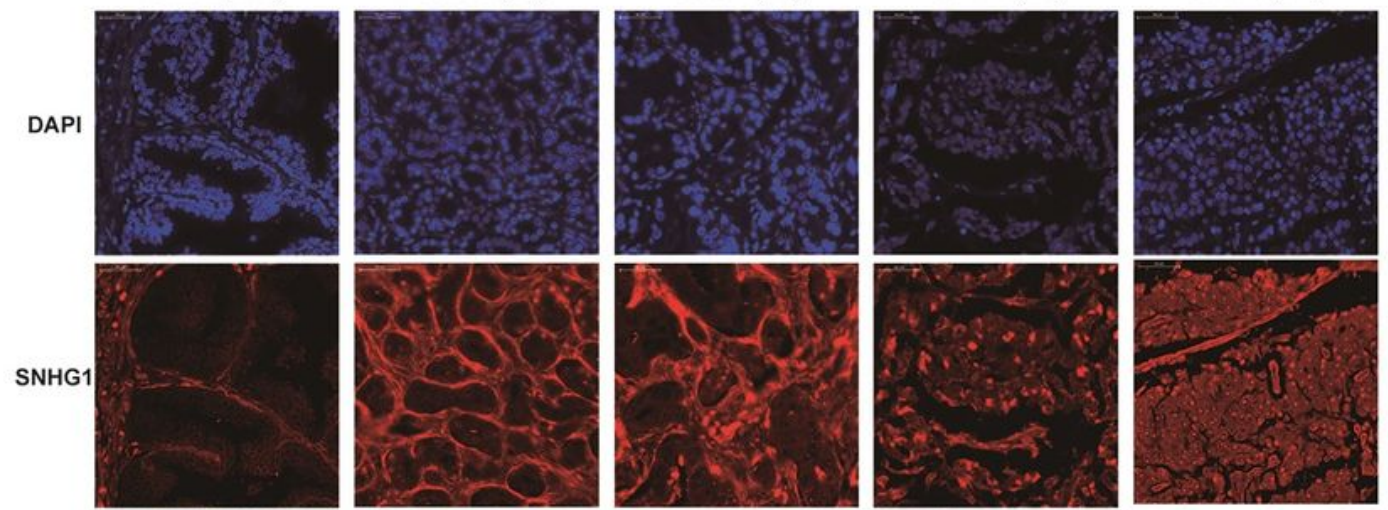

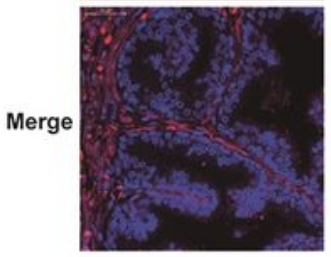

E

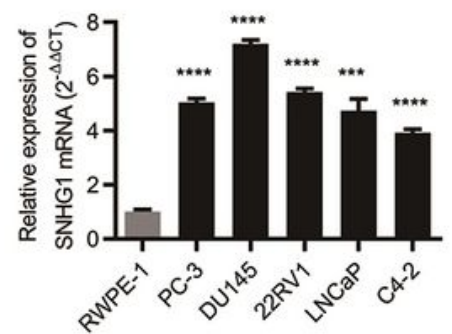

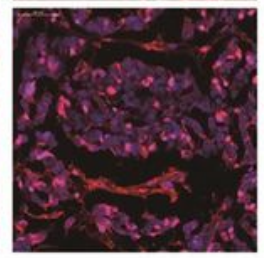

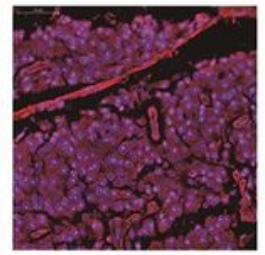

F

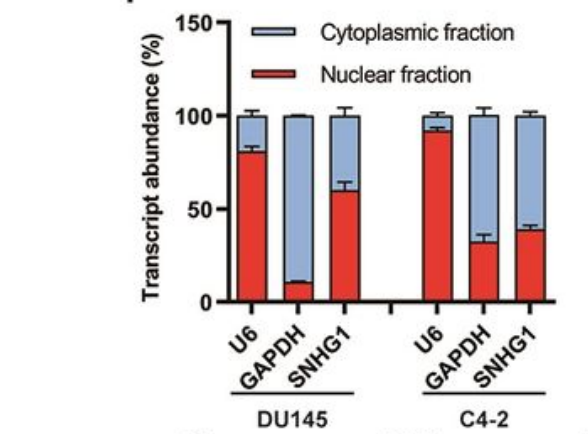

G

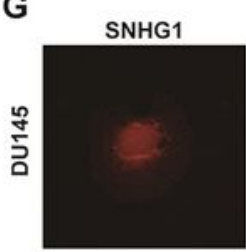

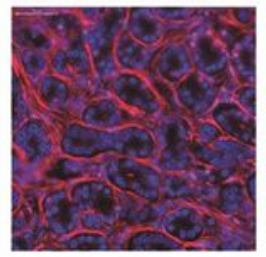

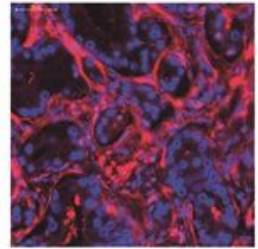

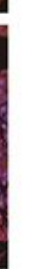
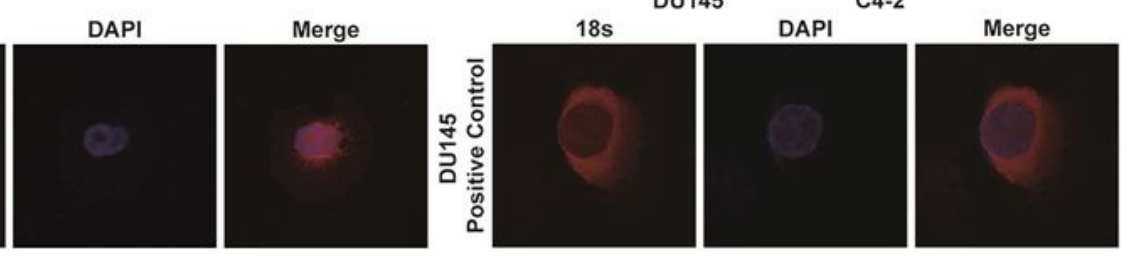

\section{Figure 1}

LncRNA SNHG1 is overexpressed in prostate cancer tissues. (A) SNHG1 is overexpressed in TCGA PCa data. (B and C) Survival analysis of SNHG1 (TCGA PRAD) showed that both overall and disease-free survival were worse in the highly expressed group. (D) FISH analysis of PCa tissues confirmed that SNHG1 was highly expressed in PCa tissues and located in the nucleus (G means Gleason grade group). (E) SNHG1 expression was analyzed by qRT-PCR in five PCa cancer cell lines (DU145, PC-3, 22RV1, 
LNCaP and C4-2), compared with the human Prostatic immortalized epithelial cells (RWPE-1). (F) Distribution of SNHG1 in PCa cells detected by fractionation of DU145 and C4-2 cells followed by qRTPCR. (G) FISH analysis of DU145 cell. The nuclei were stained with DAPI and 18S was used as a positive control for cytoplasmic staining. GS means Gleason Score. Scale bar, $20 \mu \mathrm{m}$. All data are shown as the mean \pm SD. ${ }^{*} P<0.05, * * P<0.01,{ }^{* * *} P<0.001$ and $* * * * P<0.0001$ by two-tailed Student's t-test.
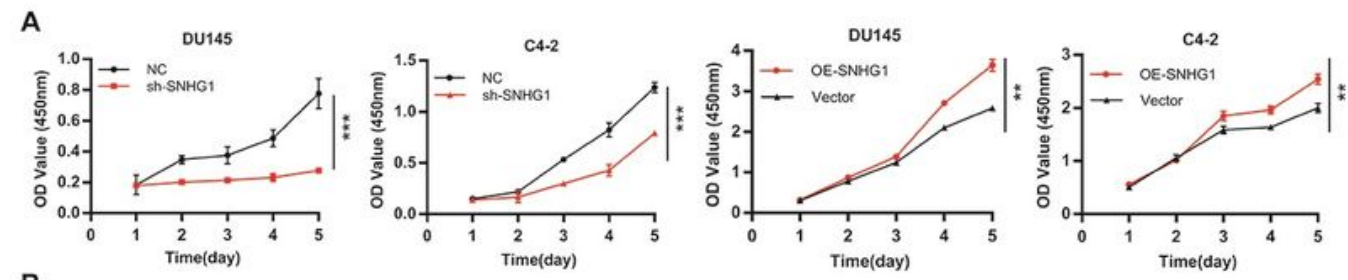

B
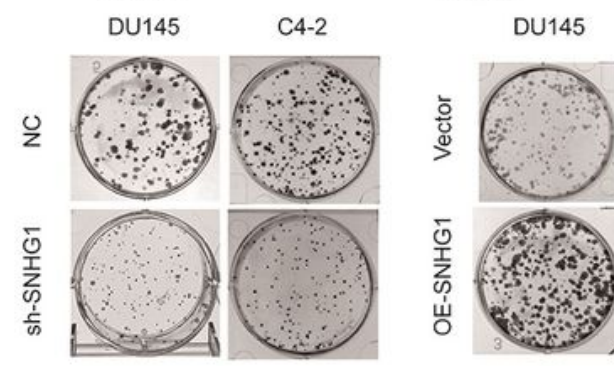

C4-2
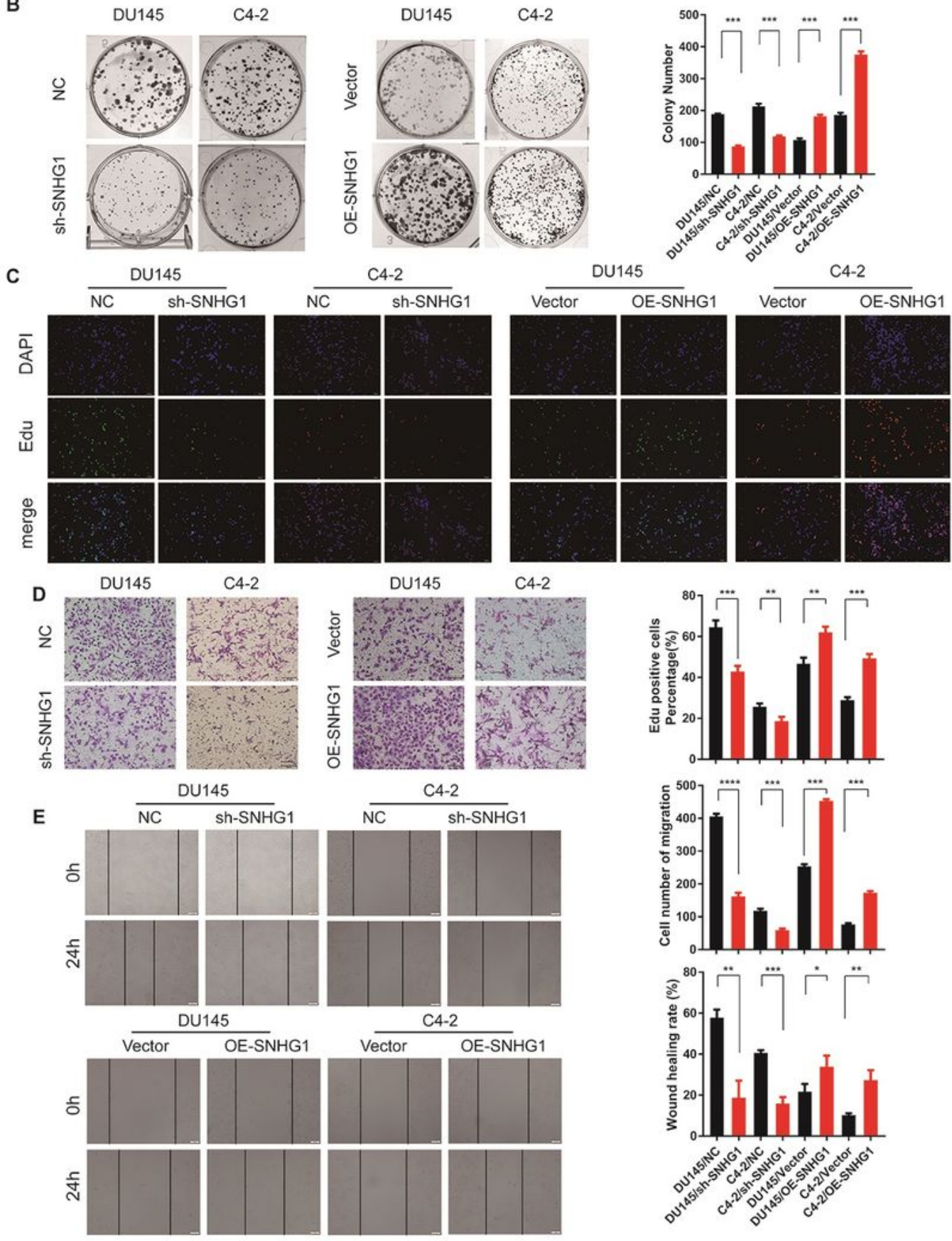

Figure 2 
LncRNA SNHG1 promotes PCa cell proliferation, migration, and invasion in vitro. (A and B) CCK-8 assay and colony-formation assay were used to determine the proliferation ability of PCa cells by knocking down or overexpressing LncRNA SNHG1. (C) Transwell assays showed that SNHG1 knockdown or overexpression could inhibit or promote PCa cell migration. (D) EdU assays were used to determine the proliferation ability of PCa cells. (E) SNHG1 knockdown attenuated cell migration in DU145 and C4-2 cells, while up-regulation of SNHG1 showed the opposite results. ${ }^{*} P<0.01, * * P<0.01,{ }^{* \star *} P<0.001$, $* * * * P<0.0001$

A

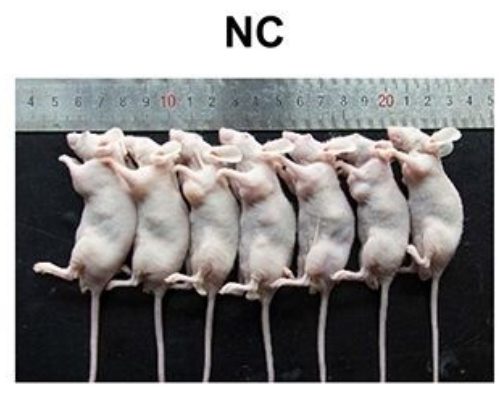

C

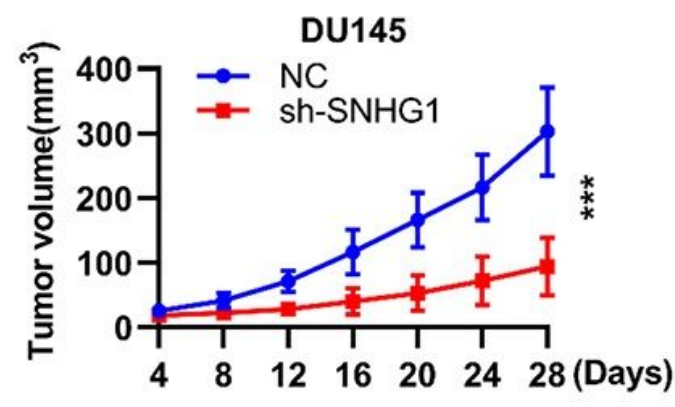

E

HE

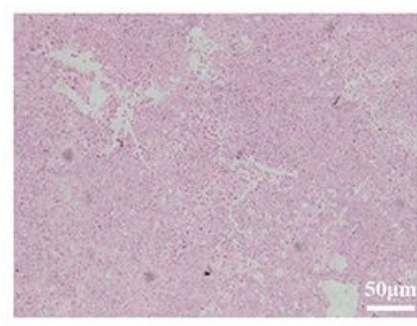

NC

sh-SNHG1

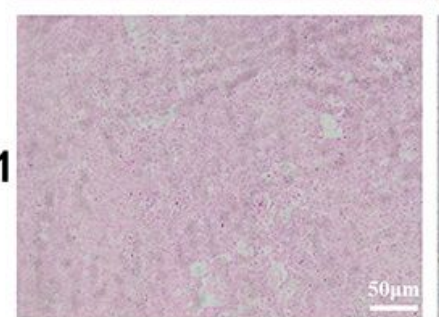

$\mathrm{Ki}-67$
sh-SNHG1

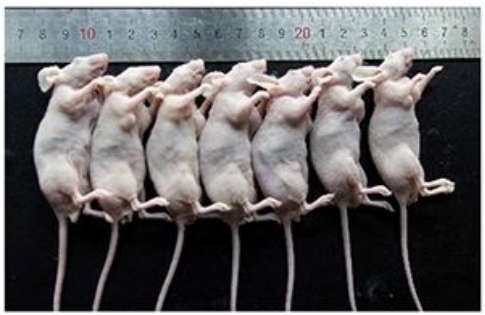

B

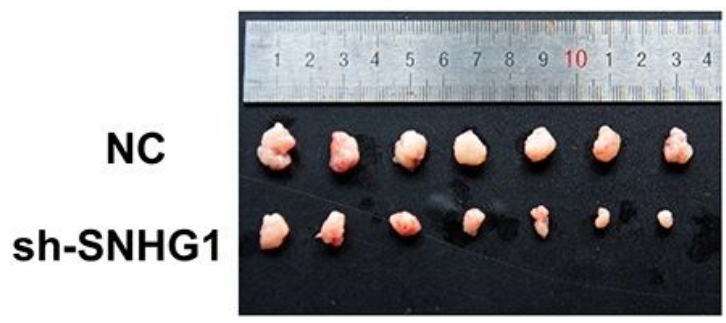

D
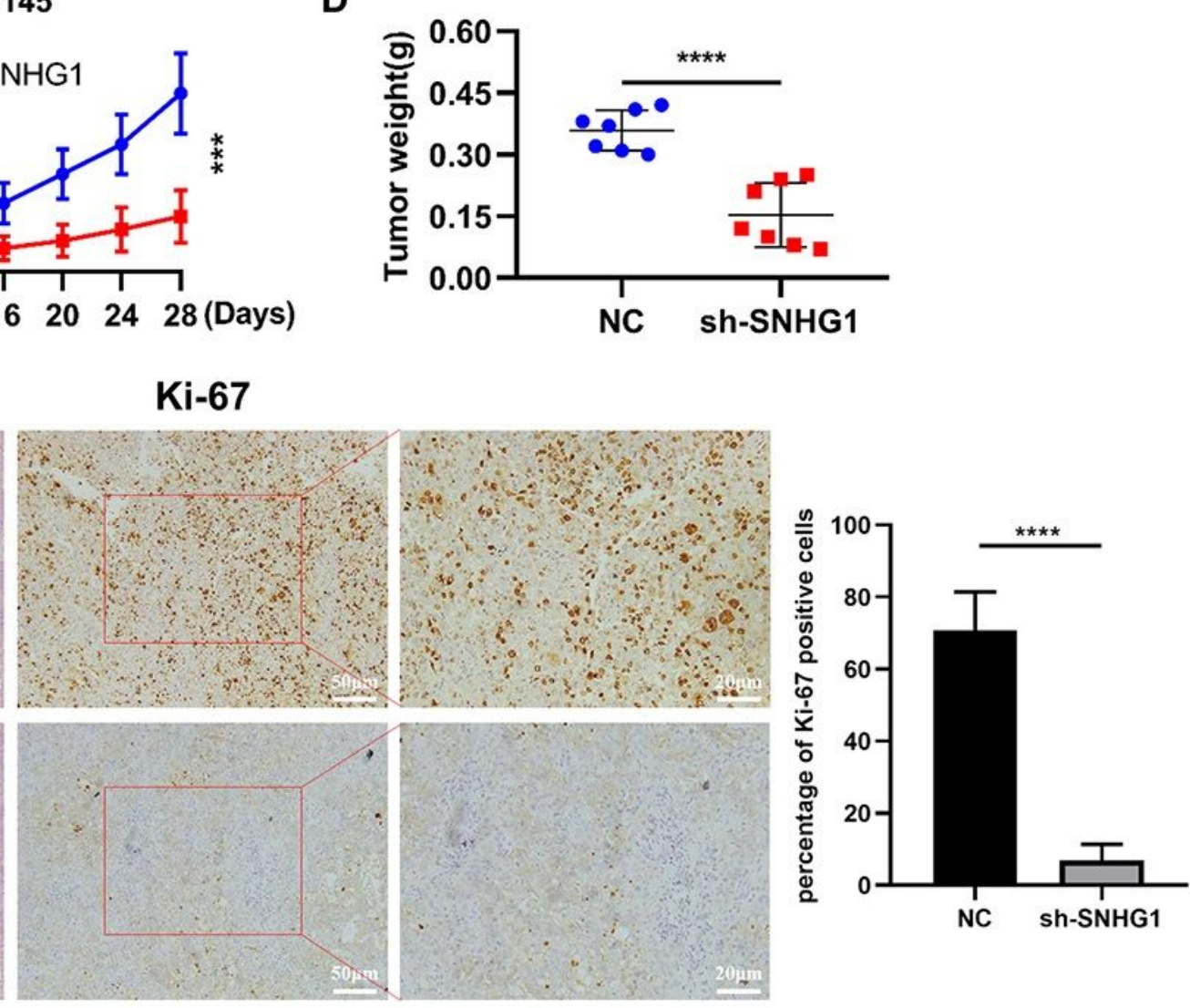

Figure 3

Subcutaneous tumor formation in nude mice confirmed that silencing SNHG1 inhibited the proliferation of PCa cells in vivo. (A-B) Negative control or sh-SNHG1 was stably transfected into DU145 cells, which 
were injected into the nude mice. (C) Tumor volumes were calculated after injection every 4 days. (D) Tumor weights were represented as means of tumor weights \pm SD. (E) IHC analyses of ki-67 confirmed that the proliferation of subcutaneous tumor was inhibited in sh-SNHG1 group. ${ }^{* \star \star} P<0.001$, ${ }^{\star \star \star *} P<0.0001$.

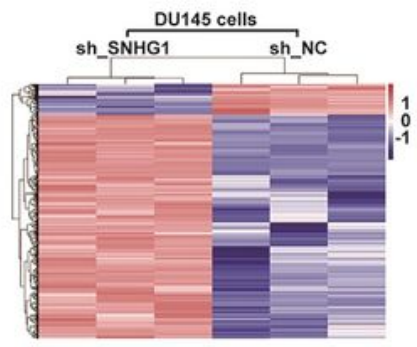

C
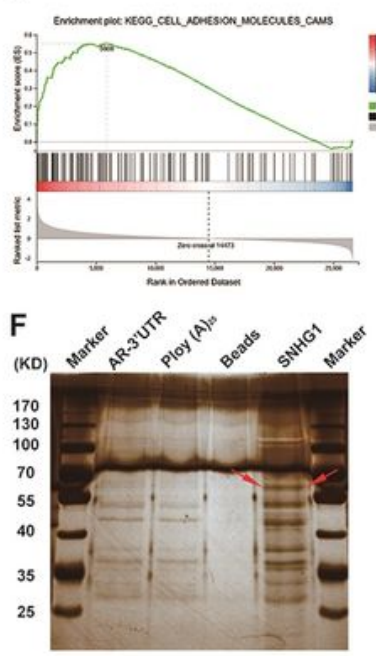

I
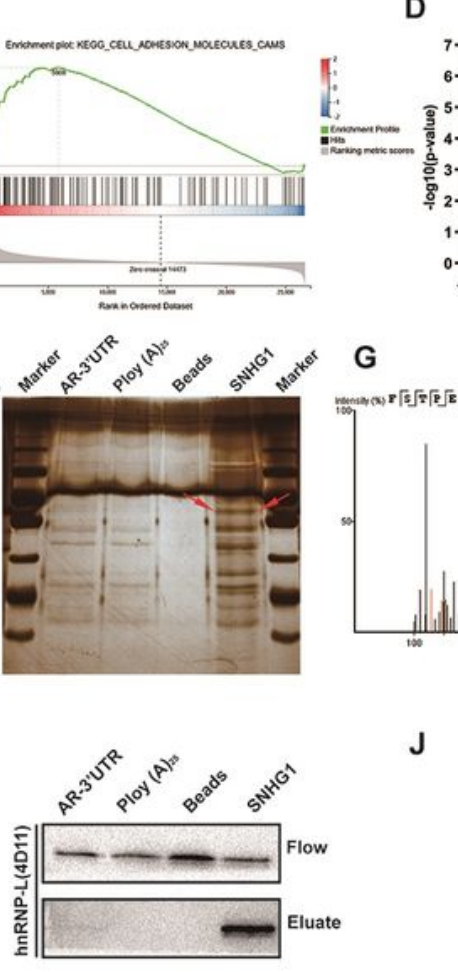

G

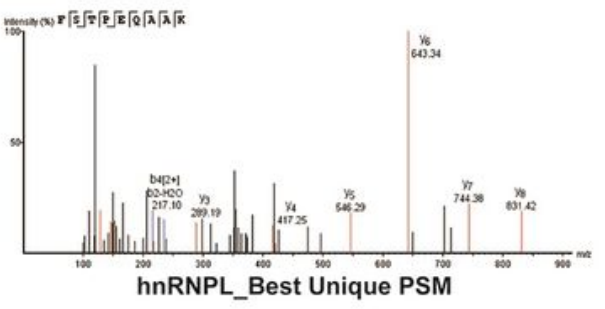

K

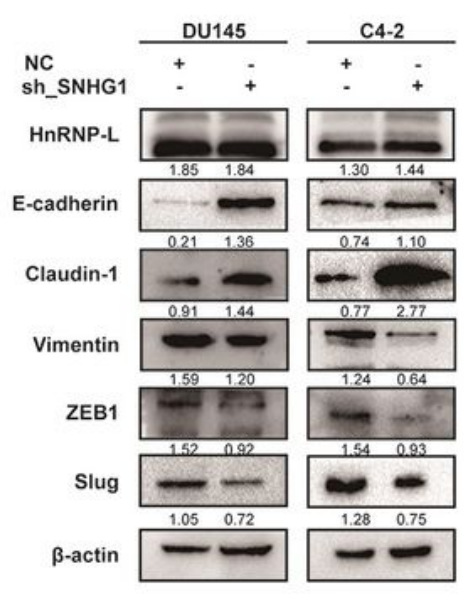

D

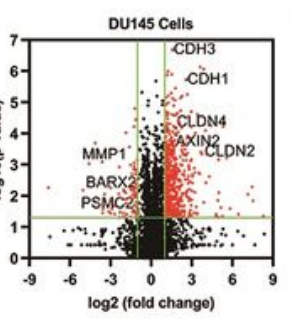

H

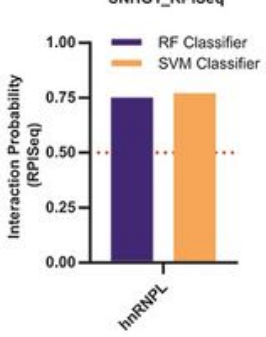

B

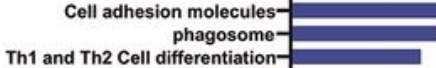

Th17 cell differentiation-

Tight junction-

Endocytosis-

ECM-receptor interaction-

E

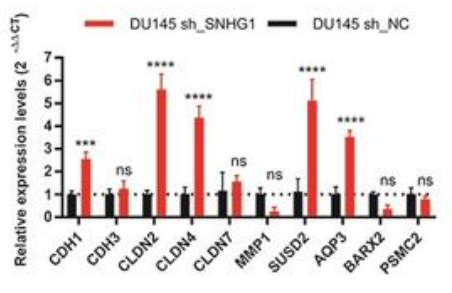

J
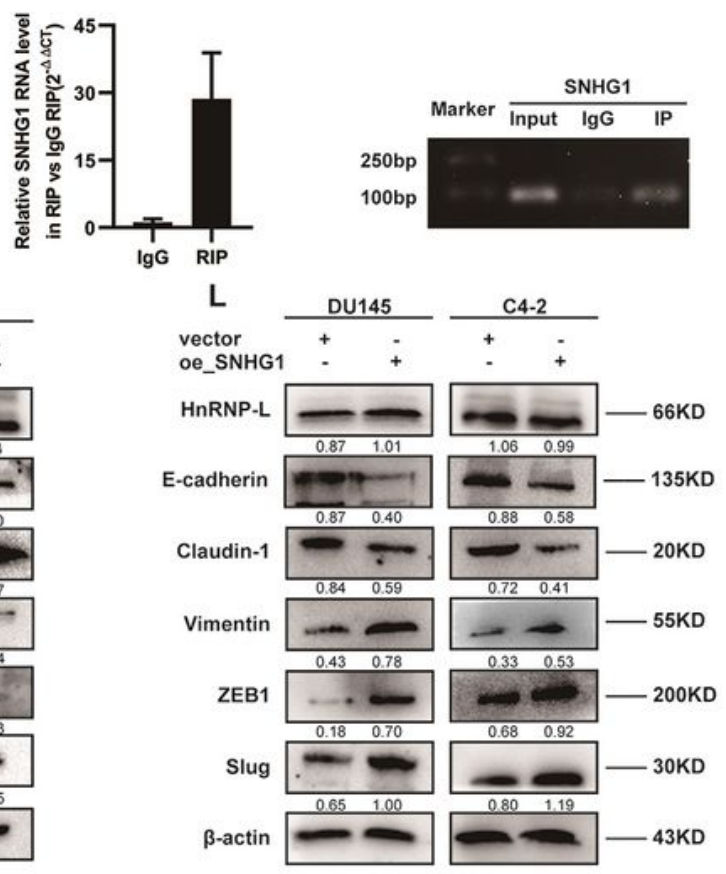

Figure 4

SNHG1 could directly bind to hnRNPL and regulate EMT. (A) Hierarchical clustering of 1045 transcripts altered ( $\geq 1.5$-fold change) in NC-treated cells and shRNA SNHG1-treated cells with three repeats. (B and 
C) KEGG analysis and differential genes GSEA enrichment analysis demonstrated that cell adhesion molecules are the potential targets of SNHG1 pathway. (D) Volcanic map analysis of differential genes. (E) The altered mRNA levels of genes were selectively confirmed by qRT-PCR in knockdown SNHG1. (F) SDS-PAGE silver staining of RNA pull-down protein samples showed a significant difference in protein band from 55 to $70 \mathrm{kDa}$. (G) Differential protein band mass spectrometry showed that the protein was hnRNPL. $(\mathrm{H})$ The interation probabilies of hnRNPL and SNHG1 were detected in silico, and the results showed that hnRNPL could well bind with SNHG1 well (RPISeq). (I) Pull-down assays showed that desthiobiotinylated SNHG1 could retrieve hnRNPL in DU145 cells by western blot(flow-through was a input control). (J) RNA immunoprecipitation revealed that hnRNPL could also specifically bind to SNHG1. ( $\mathrm{K}$ and L)The western blotting results after SNHG1 knockdown in DU145 and C4-2 cells showed that the expression level of hnRNPL did not change, while E-cad and Claudin-1 were significantly up-regulated, Vimentin, Slug and ZEB1 were significantly decreased. overexpresion of SNHG1 showed an opposite

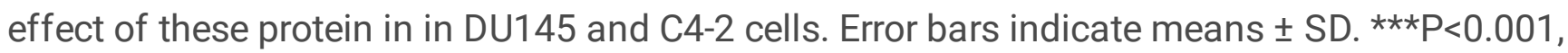
$\star \star \star * \mathrm{P}<0.0001$. 
A

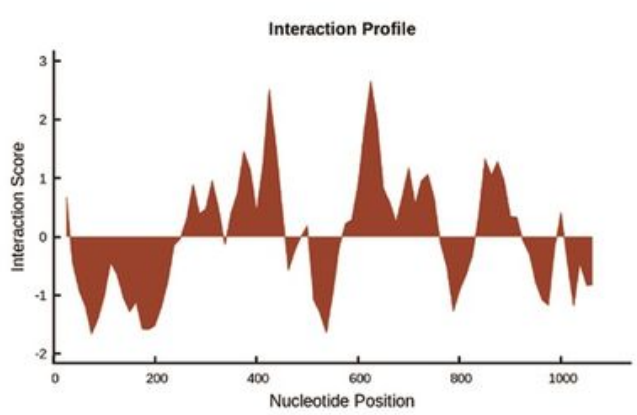

B

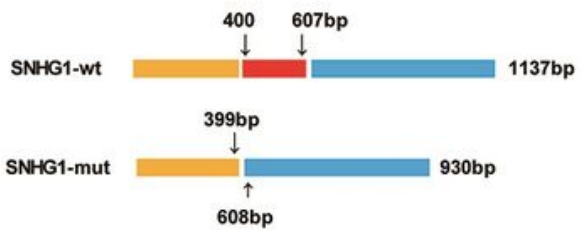

C
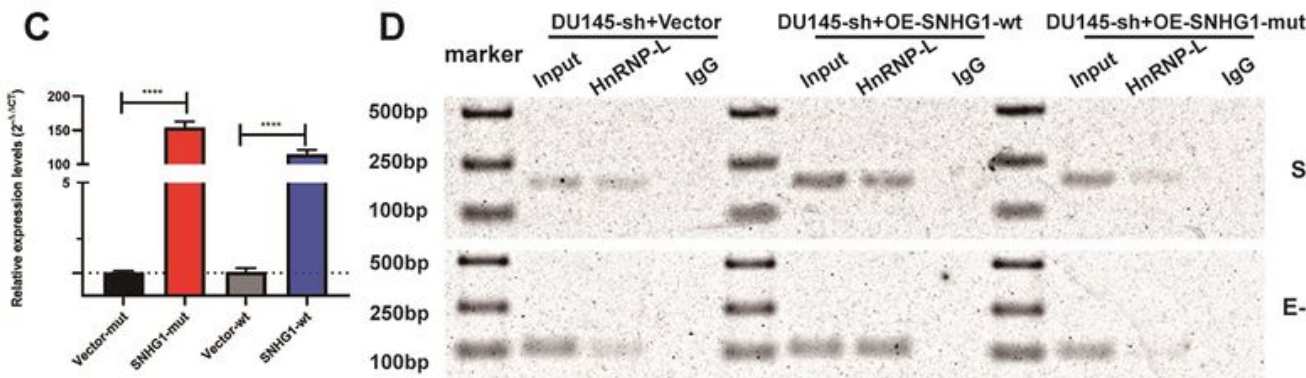

SNHG1

E-cadherin

E

DU145/sh-SNHG1
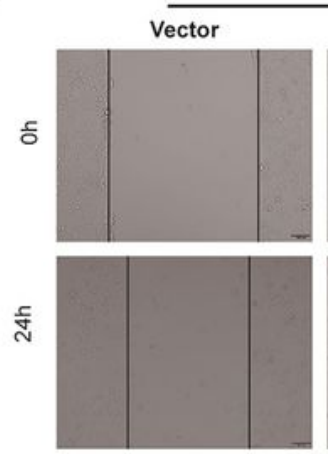
OE-SNHG1-wt OE-SNHG1-mut

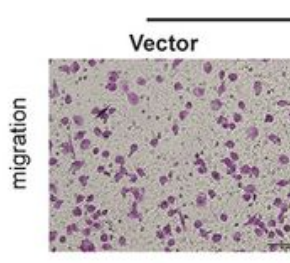

OE-SNHG1-wt

OE-SNHG1-mut
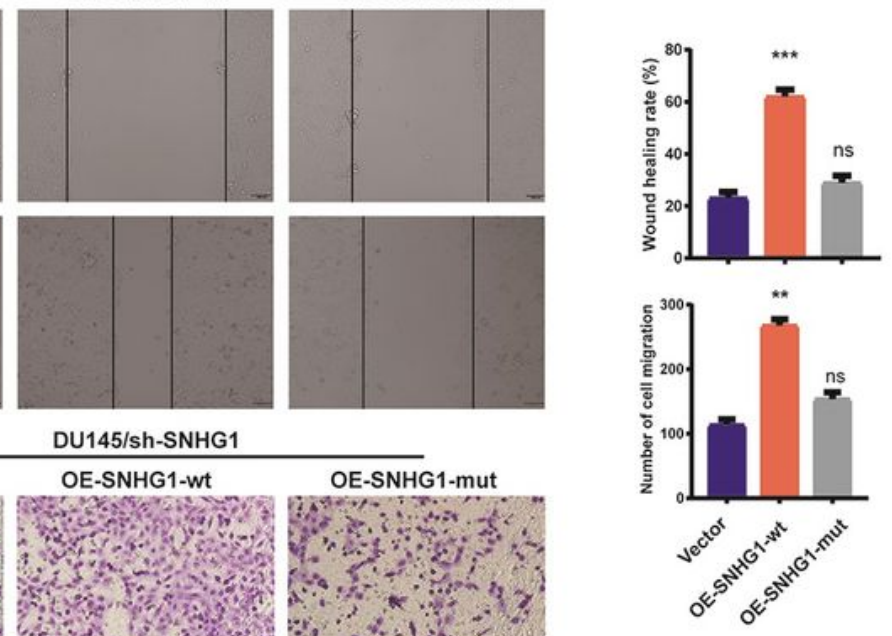

G

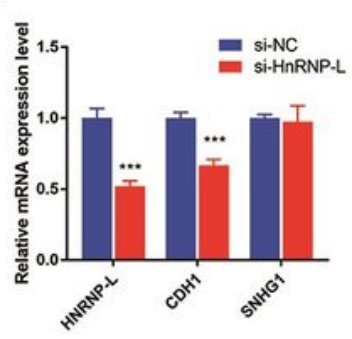

H
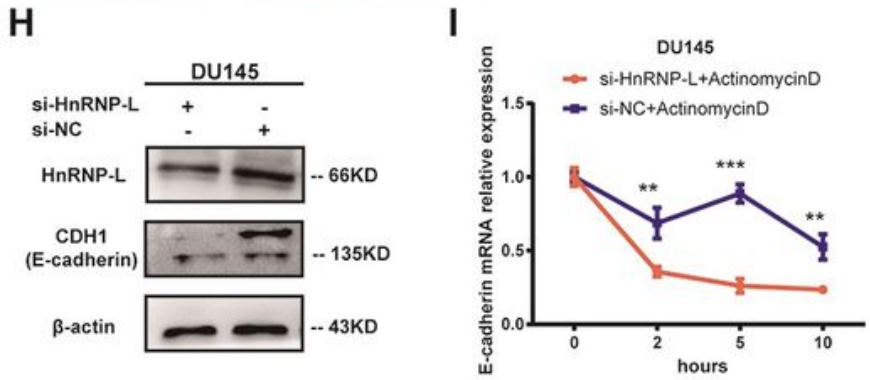

\section{Figure 5}

SNHG1/hnRNPL complex plays a vital role in PCa metastasis via stablizing CDH1 mRNA. (A) CatRAPID fragment prediction revealed that the sequence of SNHG1 at 400-600bp had a significant binding possibility with hnRNPL (http://service.tartaglialab.com/page/catra-pid_group). (B) Schematic diagram of 400-607bp truncated mutation in SNHG1. (C) Relative quantitative detection of truncated mutation and wild-type SNHG1 overexpression by qRT-PCR. (D) HnRNPL RIP confirmed that knockdown SNHG1 
reduced the binding ability of hnRNPL to $\mathrm{CDH} 1$, and the overexpression of wild-type SNHG1 could restore the binding ability, while the SNHG1-mut with poor binding ability of hnRNPL could not rescue hnRNPL to capture CDH1. (E and F) Effects of overexpression of wild type SNHG1 or mutant SNHG1 on cell migration by wound healing and migration assays. ( $G$ and $H)$ Knockdown of hnRNPL suppressed the mRNA and protein expression of CDH1(E-cadherin). (I) HnRNPL knockdown in DU145 cells downregulated $\mathrm{CDH} 1$ (E-cadherin) mRNA abundance, while ActinomycinD $(2.0 \mathrm{ug} / \mathrm{ml})$ were used to inhibit RNA synthesis. All data are shown as the mean $\pm S D$. ${ }^{*} P<0.01$, ${ }^{\star \star \star} P<0.001,{ }^{\star \star * *} P<0.0001$ by two-tailed Student's t-test. 
A

\section{DU145}

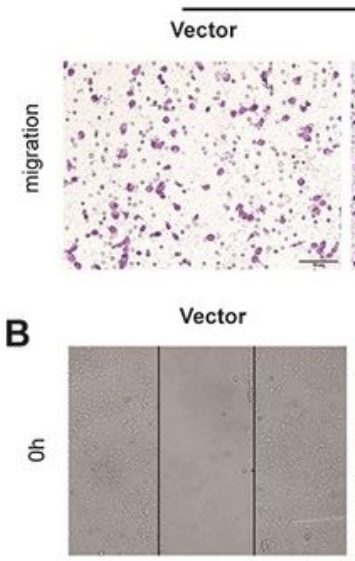

OE-SNHG1 +si-HnRNP-L

OE-SNHG

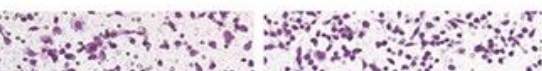

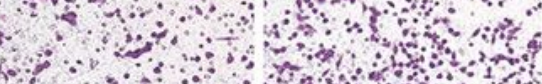

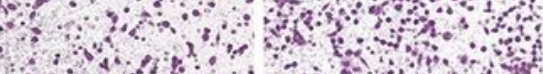

OE-SNHG1 +si-HnRNP-L

OE-SNHG1
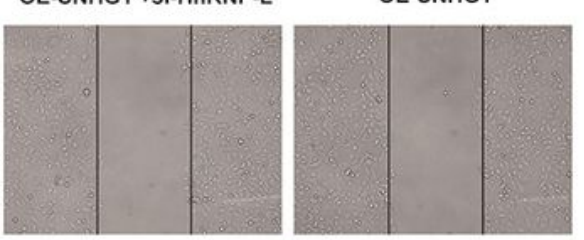

采
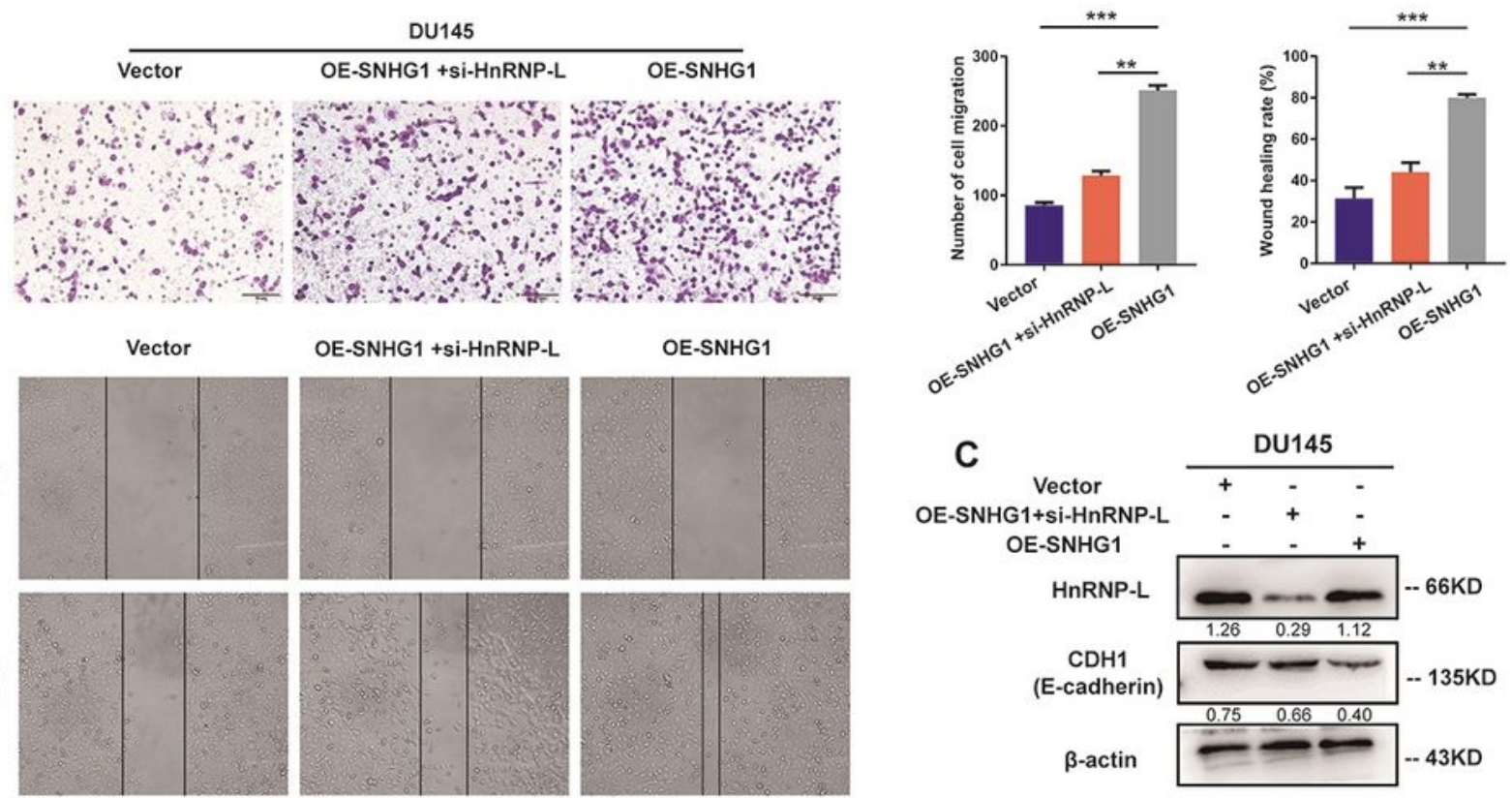

D
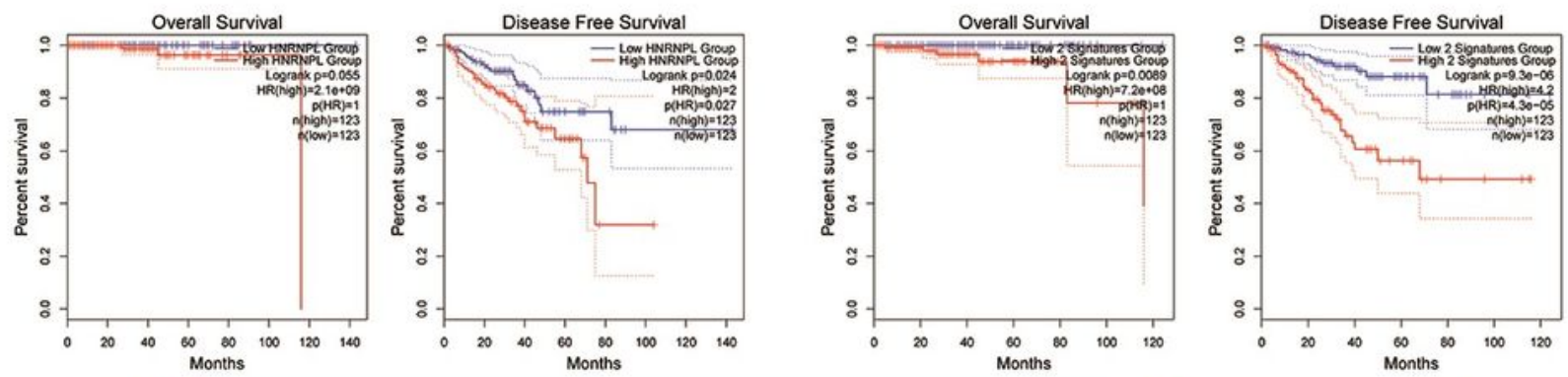

$\mathbf{F}$

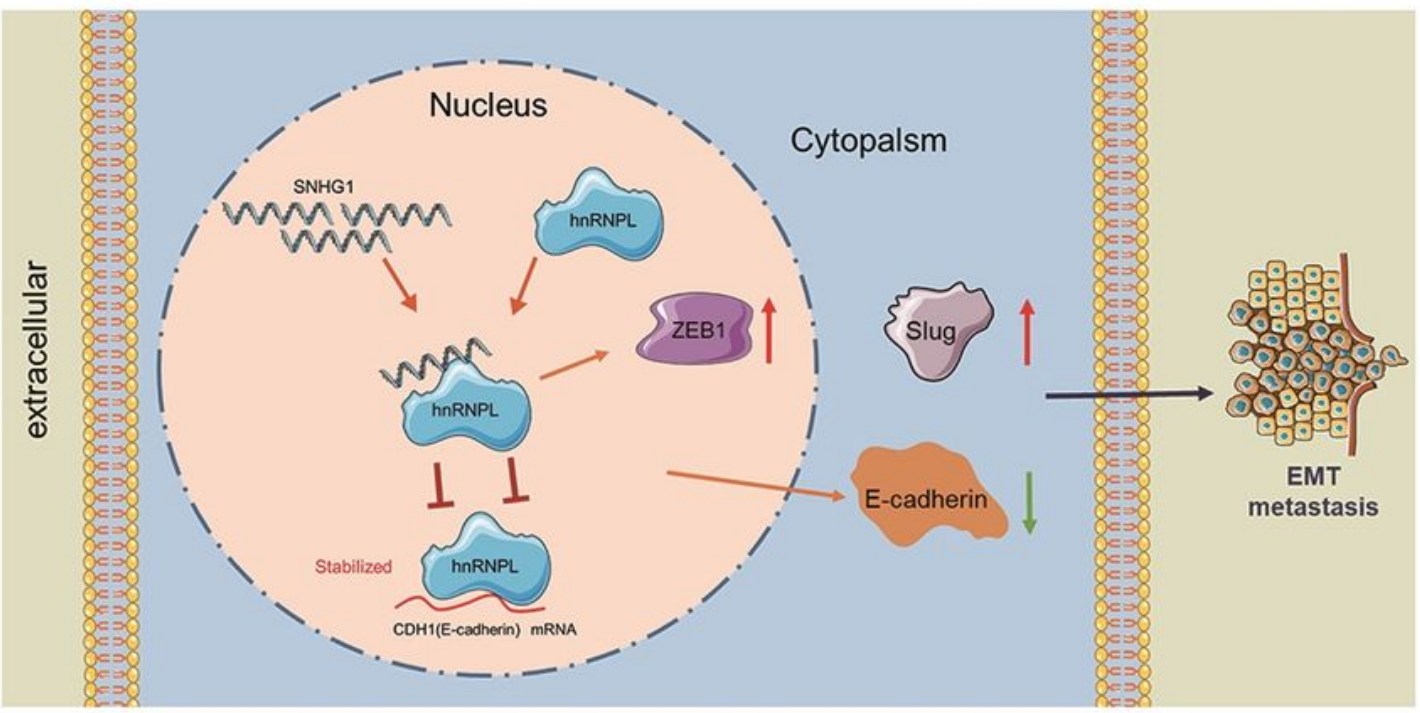

\section{Figure 6}

HnRNPL knocdown reverses the carcinogenic effects of SNHG1 promotion on cell metastasis. (A and B) HnRNPL knockdown reversed the SNHG1 overexpression-induced cell migration ability in PCa cells. (C) western blot showed that forced expression of hnRNPL reversed the SNHG1 upregulating-induced inhibition of E-cadherin protein. (D) Survival analysis revealed that PCa patients with both low SNHG1 and hnRNPL expression levels had better overall survival and disease-free survival (GEPIA, 
http://gepia.cancer-pku.cn/). (F) Schematic diagram displaying the mechanism underlying SNHG1 could competitively bind to hnRNPL leading to downregulating of E-cadherin.

\section{Supplementary Files}

This is a list of supplementary files associated with this preprint. Click to download.

- TableS1.xIsx 